Acta Crystallographica Section D

Biological Crystallography

ISSN 0907-4449

\section{S. K. Talapatra, \\ A. W. Schüttelkopf* and F. Kozielski}

Molecular Motor Laboratory, The Beatson Institute for Cancer Research, Garscube Estate, Switchback Road, Bearsden, Glasgow G61 1BD, Scotland, UK

Correspondence e-mail:

s.talapatra@beatson.gla.ac.uk,

a.schuettelkopf@beatson.gla.ac.uk

\title{
The structure of the ternary Eg5-ADP-ispinesib complex
}

The human kinesin Eg5 is responsible for bipolar spindle formation during early mitosis. Inhibition of Eg5 triggers the formation of monoastral spindles, leading to mitotic arrest that eventually causes apoptosis. There is increasing evidence that $\mathrm{Eg} 5$ constitutes a potential drug target for the development of cancer chemotherapeutics. The most advanced Eg5-targeting agent is ispinesib, which exhibits potent antitumour activity and is currently in multiple phase II clinical trials. In this study, the crystal structure of the Eg5 motor domain in complex with ispinesib, supported by kinetic and thermodynamic binding data, is reported. Ispinesib occupies the same induced-fit pocket in Eg5 as other allosteric inhibitors, making extensive hydrophobic interactions with the protein. The data for the Eg5-ADP-ispinesib complex suffered from pseudo-merohedral twinning and revealed translational noncrystallographic symmetry, leading to challenges in data processing, space-group assignment and structure solution as well as in refinement. These complications may explain the lack of available structural information for this important agent and its analogues. The present structure represents the best interpretation of these data based on extensive data-reduction, structure-solution and refinement trials.

\section{Introduction}

Proteins involved in mitosis are attractive potential targets for cancer therapy, as their inhibition may allow the specific targetting of proliferating cells (Bergnes et al., 2005; Harrison et al., 2009). Indeed, there are a number of antimitotic drugs in clinical use, all of which target microtubule (MT) dynamics. Unfortunately, however, these drugs show significant side effects, as MTs are involved in a wide variety of cellular processes aside from mitosis.

Kinesin motor proteins move along MTs in an ATPdependent manner. While they are conventionally thought to function in cellular cargo transport, a number of kinesins have been found to act during mitosis (Wordeman, 2010), making them potential targets for antimitotic drugs (Good et al., 2011). In fact, some of the mitotic kinesins appear to function exclusively during mitosis and as such they may deliver on the promise of an improved side-effect profile in anticancer therapy through inhibition of mitotic proteins.

Human Eg5 (KSP, kinesin spindle protein, KIF11), a member of the kinesin-5 family (Miki et al., 2003), is a well characterized mitotic kinesin that is required to establish a bipolar mitotic spindle. $\mathrm{Eg} 5$ forms homotetramers that can attach to neighbouring antiparallel spindle MTs and slide them against each other, thus separating the duplicated centrosomes (Kapitein et al., 2005).
Received 31 March 2012

Accepted 20 June 2012

PDB Reference: Eg5-ADPispinesib complex, $4 a p 0$. 
Loss of Eg5 function owing to RNA interference or smallmolecule inhibitors results in the formation of monoastral spindles, cell-cycle arrest and apoptosis (Blangy et al., 1995; Weil et al., 2002; Mayer et al., 1999). A number of smallmolecule inhibitors of Eg5 have been identified, including MK-0731, pyrrolotriazin-4-one-based inhibitors and the quinazolin-4-one-based ispinesib (Cox \& Garbaccio, 2010; Lad et al., 2008; Kim et al., 2006), all of which are allosteric inhibitors that bind to the unique L5 loop region of the catalytic domain. Ispinesib (also named SB-715992 or CK0238273) is a potent and selective inhibitor of $\mathrm{Eg} 5$ that is currently in multiple phase II clinical trials (Burris et al., 2011; Souid et al., 2010) and is one of the most advanced drug candidates. The importance of the quinazolin-4-one scaffold targeting $\mathrm{Eg} 5$ is further underlined by the fact that three structurally related compounds are in various stages of clinical development: SB-743921, a second-generation ispinesib analogue (Holen et al., 2011), AZD4877 (Esaki et al., 2011) and Arq621 (Chen et al., 2011). A greater understanding of the molecular details of the protein-inhibitor interactions of this class of compounds is therefore crucial.

Although an Eg5-ispinesib complex has been reported previously (Zhang et al., 2008), no coordinates or experimental data were made available, which hampers detailed analysis of this important enzyme-inhibitor interaction and the use of the complex for further structure-guided design. Here, we report the $2.6 \AA$ resolution structure of the ternary complex of the $\mathrm{Eg} 5$ motor domain in complex with $\mathrm{Mg}^{2+} \mathrm{ADP}$ and ispinesib. The structure provides a detailed overview of the interaction between ispinesib and the Eg5 motor domain and a rationale for further drug development.

\section{Methods}

\subsection{Cloning, expression and purification of Eg5}

The motor domain of human Eg5 (residues 1-368) was cloned, expressed and purified as described previously (Kaan et al., 2010).

\subsection{Steady-state ATPase-activity assay}

Steady-state basal and MT-stimulated ATPase rates were measured using the pyruvate kinase/lactate dehydrogenase linked assay (Hackney \& Jiang, 2001). The amounts of Eg5 were optimized to 80-100 $\mathrm{n} M$ for basal and $5 \mathrm{n} M$ for MTstimulated activity assays. The $\mathrm{IC}_{50}$ values for the inhibition of the basal and MT-stimulated ATPase activities of Eg5 were measured for ispinesib up to 3.0 and $1.5 \mu M$. The ATP concentration was fixed at $1 \mathrm{~m} M$ and MTs were used at $2 \mu M$ where applicable. Data were analysed using Kaleidagraph v.4.0 (Synergy Software). ATPase measurements were performed at $298 \mathrm{~K}$ using a 96-well Sunrise photometer (Tecan, Mannesdorf, Switzerland). MTs were prepared from lyophilized tubulin (tebu-bio catalogue No. 027T240-B) as described previously (Kozielski et al., 2007).

\subsection{Isothermal titration microcalorimetry (ITC)}

ITC was performed as described previously by Sheth $e t$ al. (2009) with minor modifications. Purified Eg5 was subjected to gel-filtration chromatography in buffer $A$ ( $20 \mathrm{~m} M$ PIPES $\mathrm{pH} 6.8,300 \mathrm{~m} M \mathrm{NaCl}, 2 \mathrm{~m} M \beta$-mercaptoethanol) to remove excess ATP and was then dialyzed overnight against buffer $A$ supplemented with $0.5 \mathrm{~m} M$ ADP and $5 \mathrm{mM} \mathrm{MgCl}_{2}$. The protein was diluted to a final concentration of $20 \mu M$ with dialysis buffer. The protein concentration was then verified by absorption measurements at $280 \mathrm{~nm}$ employing an experimental extinction coefficient determined using Eg5 denatured in $6.7 M$ guanidine hydrochloride with $20 \mathrm{~m} M$ phosphate $\mathrm{pH}$ 7.0 and including the absorption of ADP. Finally, 1\% DMSO was added to the protein solution. The inhibitor was prepared in $100 \%$ DMSO and then diluted in dialysis buffer to a final concentration of $250 \mu M$ ispinesib with $1 \%$ DMSO. All solutions were centrifuged for 5-10 min at room temperature prior to loading of the samples into the ITC cell. ITC experiments were performed with a Microcal VP-ITC titration calorimeter (Microcal Inc., North Hampton, Massachusetts, USA). All titrations were carried out at $298 \mathrm{~K}$ with a stirring speed of $350 \mathrm{rev} \mathrm{min}^{-1}$. A total of 26 injections were performed per titration; the first injection of $5 \mu \mathrm{l}$ was followed by 25 injections of $10 \mu \mathrm{l}$ with a gap of $240 \mathrm{~s}$ between them. The heat of dilution was subtracted prior to data analysis. The thermodynamic parameters $n$ (stoichiometry), $K_{\mathrm{a}}$ (association constant) and $\Delta H$ (enthalpy change) were obtained through fitting of the experimental data using the single-site binding model of the Origin software package (v.7.0); the free energy of binding $(\Delta G)$ and entropy change $(\Delta S)$ were then calculated from the fitted values. For each experiment, at least two independent titrations were performed which were analysed independently. The resulting thermodynamic values were then averaged.

\subsection{Crystallization of the Eg5-ispinesib complex}

Purified Eg5 at $10 \mathrm{mg} \mathrm{ml}^{-1}$ was mixed with $1 \mathrm{mM} \mathrm{Mg}{ }^{2+}$ ATP and then incubated with ispinesib at a final concentration of $1 \mathrm{~m} M$ for $2 \mathrm{~h}$ at $277 \mathrm{~K}$; the sample was then centrifuged at $14000 \mathrm{~g}$ for $5 \mathrm{~min}$ at $277 \mathrm{~K}$ to pellet undissolved inhibitor. Initial crystals of the complex were obtained at $277 \mathrm{~K}$ by vapour diffusion in sitting or hanging drops consisting of $200 \mathrm{nl}$ protein-inhibitor complex and $200 \mathrm{nl}$ reservoir solution equilibrated against a reservoir consisting of $0.1 M$ Tris $\mathrm{pH} 8.5$, $0.02 \mathrm{M} \mathrm{MgCl}_{2}, 20 \%(w / v)$ polyethylene glycol (PEG) 8000 . Crystals were subsequently grown using identical conditions in 24-well plates (Linbro, Hampton Research) using drops consisting of $1 \mu \mathrm{l}$ protein solution and $1 \mu \mathrm{l}$ reservoir solution and were improved by streak-seeding to generate crystals with a rectangular plate morphology that were suitable for data collection. Prior to data collection, crystals were immersed in cryoprotectant solution [0.12 $M$ Tris pH 8.5, $0.024 \mathrm{M} \mathrm{MgCl}_{2}$, $24 \%(w / v)$ PEG $8000,15 \%$ glycerol] and flash-cooled in liquid nitrogen. 
Table 1

Data-collection and structure-refinement statistics for the Eg5-ispinesib complex.

Values in parentheses pertain to the highest resolution shell of $0.15 \AA$. Ramachandran plot statistics were obtained with MolProbity (Chen et al., 2010).

\begin{tabular}{ll}
\hline Unit-cell parameters $\left(\AA{ }^{\circ}\right)$ & $\begin{array}{c}a=64.7, b=112.6, c=106.9 \\
\beta\end{array}$ \\
Space group & $P 2_{1}$ \\
Molecules per asymmetric unit & 4 \\
Resolution range $(\AA)$ & $30.0-2.6$ \\
Total reflections & 134457 \\
Unique reflections & 45511 \\
Completeness $(\%)$ & $95.7(91.0)$ \\
Multiplicity & $3.0(2.7)$ \\
$R_{\text {merge }}(\%)$ & $6.9(62.2)$ \\
$\langle I / \sigma(I)\rangle$ & $13.3(2.0)$ \\
$R_{\text {work }} / R_{\text {free }}(\%)$ & $20.4 / 25.3$ \\
Wilson $B\left(\AA^{2}\right)$ & 59.9 \\
Average $B\left(\AA^{2}\right)$ & \\
Overall & 57.7 \\
Protein & 58.2 \\
Solvent & 48.1 \\
ADP & 33.8 \\
Ispinesib & 44.9 \\
R.m.s.d. bond lengths $(\AA)$ & 0.016 \\
R.m.s.d. bond angles $\left({ }^{\circ}\right)$ & 1.47 \\
Ramachandran plot statistics $(\%)$ & \\
Favoured & 98.1 \\
Allowed & 1.9 \\
Outliers & 0 \\
\hline
\end{tabular}

\subsection{Data collection, structure determination, refinement and model quality}

Diffraction data were collected at $100 \mathrm{~K}$ on beamline I02 at Diamond Light Source. Data were processed and scaled to $2.6 \AA$ resolution using XDS/XSCALE (Kabsch, 2010), then truncated and further processed with the $C C P 4$ suite of programs (Winn et al., 2011). The structure of the Eg5-ispinesib complex was solved in space group $P 2_{1}$ by molecular replacement with MOLREP using the Eg5 tetramer of PDB entry $2 \mathrm{gm} 1$ as the search model (Kim et al., 2006). Twinning analysis was carried out with phenix.xtriage from the PHENIX suite (Adams et al., 2002). Iterative improvement of this structure then proceeded through cycles of model building with Coot (Emsley \& Cowtan, 2004) and refinement using PHENIX or REFMAC5 (Murshudov et al., 2011), resulting in a final model with an $R_{\text {free }}$ of $25.3 \%$ and overall reasonable geometry. Coordinates and dictionaries for ispinesib were obtained from the Dundee PRODRG server (Schüttelkopf \& van Aalten, 2004). Crystallographic statistics are given in Table 1. Coordinates and structure factors have been deposited in the Worldwide Protein Data Bank (PDB entry 4ap0). In the Ramachandran plot, $98.1 \%$ of the residues are in preferred regions, $1.9 \%$ of the residues are in allowed regions and there are no outliers (as calculated by MolProbity; Chen et al., 2010). Plots of per-residue real-space correlation coefficients (calculated with SFCHECK; Vaguine et al., 1999) and B factors are shown in Fig. 1.

\section{Results and discussion}

\subsection{Biochemical and biophysical investigation of ispinesib binding}

While it has been well established that ispinesib inhibits the MT-stimulated Eg5 activity with low nanomolar affinity $\left(\mathrm{IC}_{50}=5.0 \pm 0.5 \mathrm{n} M\right.$; Sheth et al., 2009), we were interested in determining whether the same holds true in the absence of MTs in order to obtain an indication of whether Eg5-ispinesib

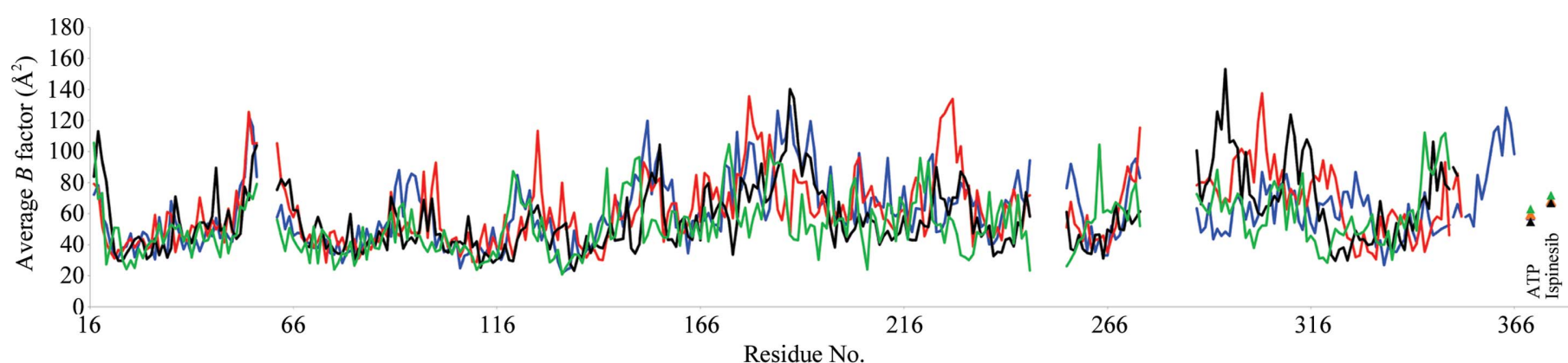

(a)

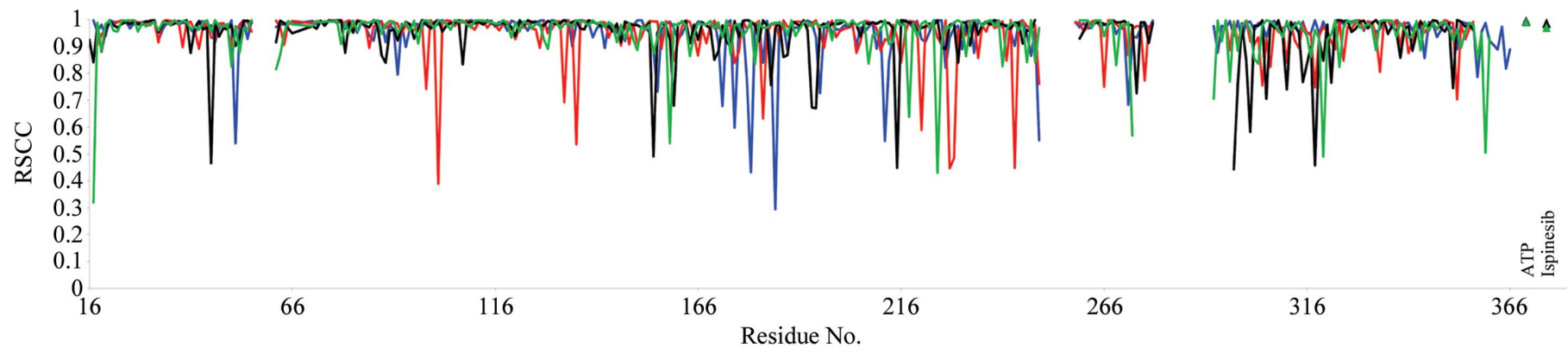

(b)

Figure 1

Plots of per-residue average $B$ factors ( $a$ ) and real-space correlation coefficients (b) for chains $A$ (blue), $B$ (red), $C$ (black) and $D$ (green). The real-space correlation coefficient (RSCC) was calculated with SFCHECK (Vaguine et al., 1999) using a $\sigma_{\mathrm{A}}$-weighted $2 F_{\mathrm{o}}-F_{\mathrm{c}}$ map. 
cocrystallization (which precludes the presence of MTs) would be likely to succeed in our hands (Table 2, Figs. $2 a$ and $2 b$ ). While the $\mathrm{IC}_{50}$ estimate obtained for MT-stimulated ATPase activity $\left(\mathrm{IC}_{50, \mathrm{MT}}=3.0 \pm 0.4 \mathrm{n} M\right)$ agrees well with the literature data, in the absence of MTs the $\mathrm{IC}_{50}$ of ispinesib increases by one order of magnitude to $32.8 \pm 0.5 \mathrm{n} M$. Given the different protein concentrations used in the assays, it is not uncommon for tight-binding inhibitors to appear less potent against the basal compared with the MT-stimulated ATPase activity of kinesins. However, this drop in affinity should not, and indeed does not, impede complex crystallization. Sheth et

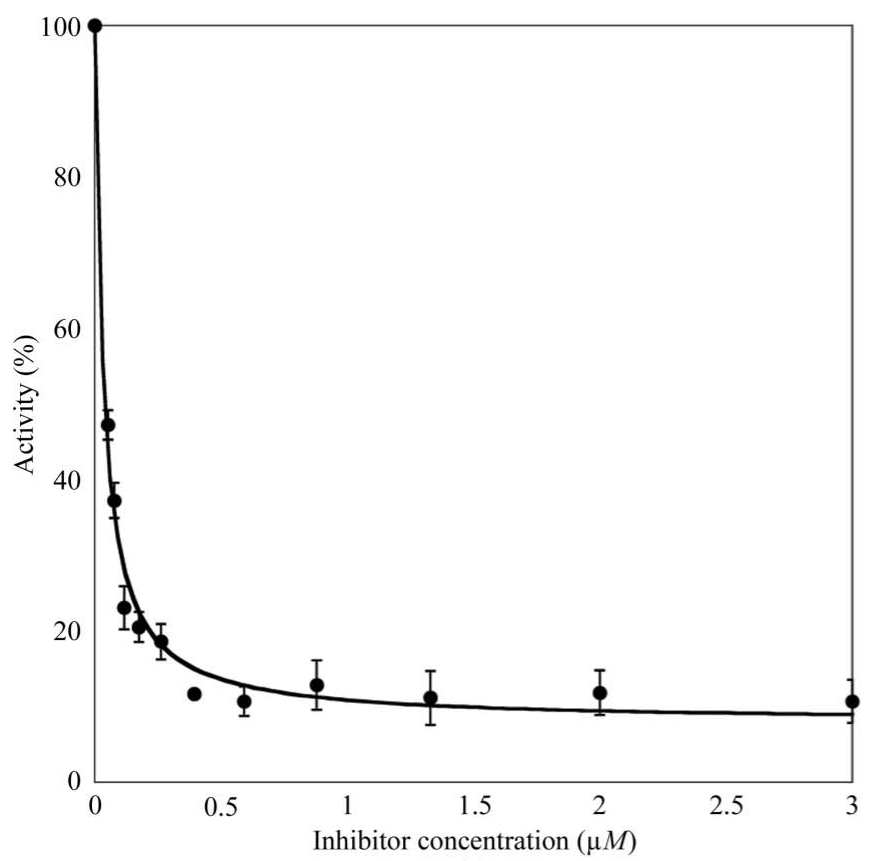

(a)

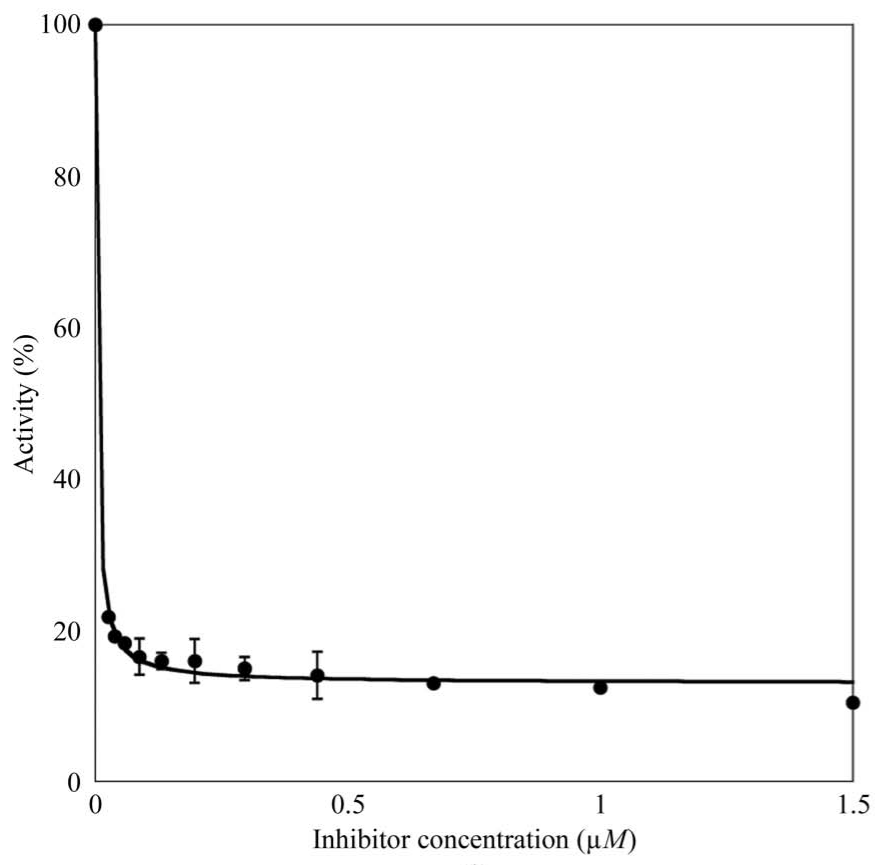

(b)
Table 2

Inhibition of Eg5 ATPase activity by ispinesib.

\begin{tabular}{lll}
\hline & Basal & MT-stimulated \\
\hline $\mathrm{IC}_{50}(\mathrm{n} M)$ & $32.8 \pm 0.5$ & $3.0 \pm 0.4$ \\
\hline
\end{tabular}

al. (2009) also performed microcalorimetric binding studies on the Eg5-ispinesib system (now in the absence of MTs), which yielded dissociation constants of less than $10 \mathrm{n} M$. To further investigate this discrepancy, we repeated the ITC experiment on the binding of ispinesib to $\mathrm{Eg} 5$ (Table 3, Fig. 2c). Our calorimetric measurements show that even in the absence of MTs ispinesib is a tight-binding Eg5 ligand $\left(K_{\mathrm{d}}<10 \mathrm{n} M\right)$, which is in agreement with the data of Sheth et al. (2009).

\subsection{Overall structure}

The structure of the Eg5-ispinesib complex was solved at $2.6 \AA$ resolution and refined to an $R_{\text {free }}$ of $25.3 \%$ with four protein molecules in the asymmetric unit. Depending on the molecule, the N-terminal $15-17$ residues as well as the C-terminal 2-9 residues of $\mathrm{Eg} 5_{1-368}$ are disordered, as are a number of loops; in particular, loops L10 and L11 (Fig. 3) are absent from all four molecules. Aside from this, the four independent complexes in the asymmetric unit are mostly

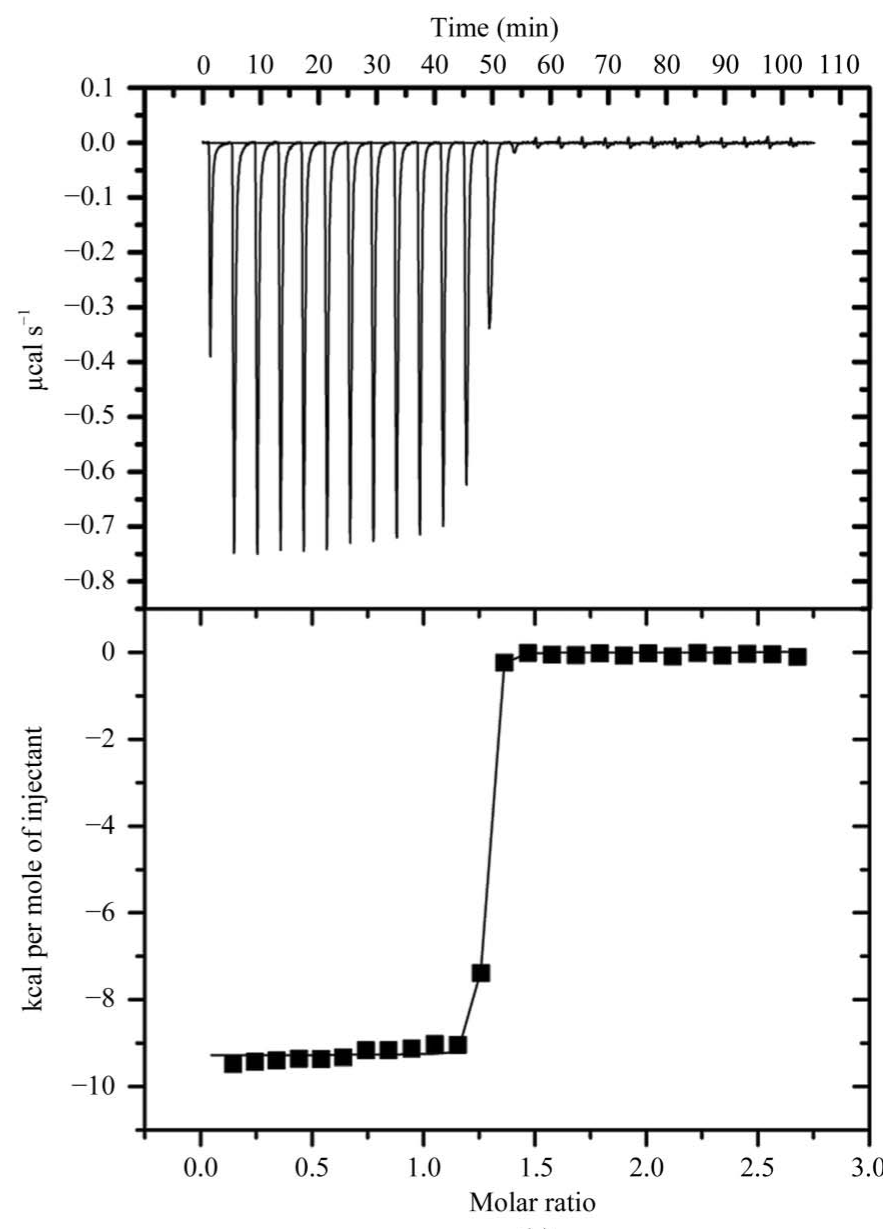

(c)

Figure 2

Characterization of the inhibition of Eg5 by ispinesib. Inhibition of the (a) basal and (b) MT-stimulated ATPase activity of Eg5. (c) Raw (top) and integrated (bottom) ITC data demonstrating saturable exothermic evolution of heat upon sequential additions of ispinesib to Eg5. $1 \mathrm{cal}=4.184 \mathrm{~J}$. 
Table 3

Thermodynamic data for the binding of ispinesib to Eg5-ADP.

Owing to the tight binding of ispinesib, the measured apparent $K_{\mathrm{d}}$ can only be treated as an upper limit. $1 \mathrm{cal}=4.186 \mathrm{~J}$.

\begin{tabular}{llll}
\hline$n$ & $K_{\mathrm{d}}^{\mathrm{app}}(\mathrm{n} M)$ & $\Delta H\left(\mathrm{kcal} \mathrm{mol}^{-1}\right)$ & $T \Delta S^{\mathrm{app}}\left(\mathrm{kcal} \mathrm{mol}^{-1}\right)$ \\
\hline $1.270 \pm 0.003$ & $\leq 10$ & $-9.08 \pm 0.04$ & $2.81 \pm 0.23$ \\
\hline
\end{tabular}

similar, with pairwise superpositions giving r.m.s. deviations of around $0.6 \AA$ for $\sim 300 \mathrm{C}^{\alpha}$ atoms. For the sake of clarity, the further discussion will thus focus only on chain $B$ unless stated otherwise.

The present structure conforms to the canonical kinesin motor domain fold with an eight-stranded $\beta$-sheet sandwiched between three major $\alpha$-helices on either side (Figs. $3 a$ and $3 b$ ). It shows one molecule of $\mathrm{Mg}^{2+} \mathrm{ADP}$ bound in the nucleotidebinding pocket with the magnesium coordinated by the $\beta$-phosphate, the side-chain hydroxyl of Thr112 and three water molecules, resulting in an octahedral geometry with one disordered ligand (presumably bulk solvent). Ispinesib occupies the inhibitor-binding pocket formed by helix $\alpha 2$, loop L5 and helix $\alpha 3$ (Fig. 3a).

Comparison with apo Eg5 (PDB entry 1ii6; Turner et al., 2001) shows that the region around the inhibitor-binding pocket undergoes major conformational changes on ispinesib binding. Additional changes extend towards the other end of the motor domain, bringing about larger conformational changes in the switch II cluster (helix $\alpha 4$, loop L12 and helix $\alpha 5)$ and the neck-linker region (Yan et al., 2004). All four molecules in the asymmetric unit depict the final ispinesibbound state. Helix $\alpha 4$ rotates and moves by around $7 \AA$ in the inhibitor-bound state compared with the native structure. The anticlockwise rotation and shift of helix $\alpha 4$ rearranges the switch II cluster and opens up space enabling the neck-linker to dock to the motor domain (Figs. $3 b$ and $3 c$ ). This shows that ispinesib brings about structural changes in the $\mathrm{Eg} 5$ catalytic domain, in agreement with published biochemical data (Lad et al., 2008).

\subsection{Ispinesib binding to Eg5 and comparison with other allosteric inhibitors}

Ispinesib is buried in the allosteric site and displays numerous interactions with residues of the inhibitor-binding pocket (Fig. 4a; Zhang et al., 2008). At the resolution obtained for this structure, we did not observe any ordered water molecules in close proximity to the inhibitor. The benzyl moiety of the ligand is buried deeply in the hydrophobic part of the pocket, where it stacks with the Pro137 ring and makes an edge-to-face interaction with the side chain of Trp127, as well as hydrophobic interactions with the side chains of Tyr211 and Leu214. In addition, the benzyl group also forms an intramolecular edge-to-face stacking interaction with the $p$-toluyl moiety of the inhibitor, which in turn stacks extensively with the mostly flat protein backbone of Glu118/Arg119 and also interacts with parts of the side chains of Arg119, Trp127 and Asp130. The isopropyl group of ispinesib is only partly buried between the side chains of Tyr211 and Leu214

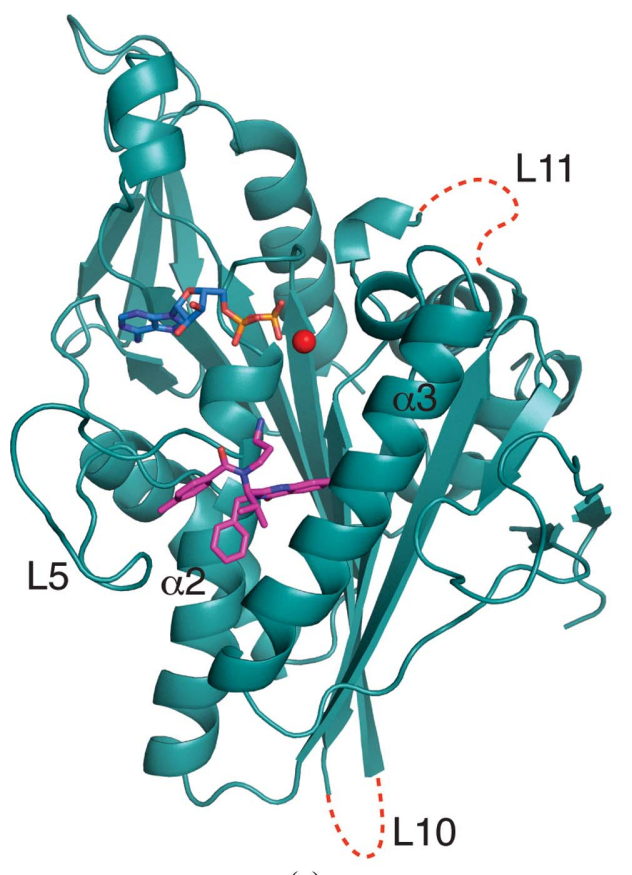

(a)

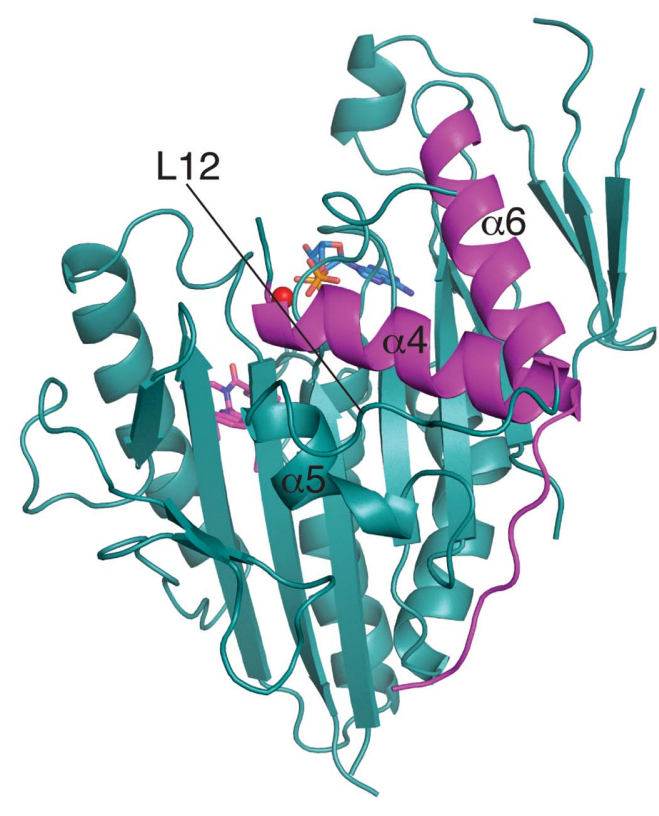

(b)

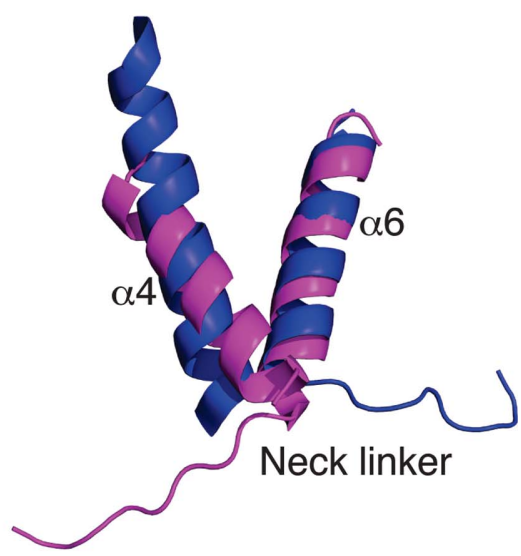

(c)

Figure 3

Overall structure of the ADP-Eg5-ispinesib ternary complex (chain B). (a) Front view of the Eg5 motor domain in complex with $\mathrm{Mg}^{2+}$ (red), $\mathrm{ADP}$ (blue) and ispinesib (magenta); red dotted lines indicate the locations of the disordered loops L10 and L11, and selected secondary-structure elements and loops are labelled. (b) Back view of the Eg5 motor domain with $\alpha 4$ of the switch II cluster, the neck-linker region as well as the preceding $\alpha 6$ helix highlighted in magenta. (c) Detailed view of helices $\alpha 4$ and $\alpha 6$ as well as the neck-linker region of the Eg5-ispinesib complex (magenta) superimposed on the apo Eg5 structure (blue; PDB entry 1ii6; Turner et al., 2001). 
as well as the backbone of the latter residue and Glu215. The chlorine substituent of the 7-chloro-3,4-dihydro-4-oxo3-(phenylmethyl)-2-quinazolinyl moiety sits in another mostly hydrophobic pocket formed by the backbone of Gly217 and the side chains of Leu160, Leu171 and Arg221. In three of the four chains $(B / C / D)$ the primary amine of ispinesib is oriented towards the ADP-binding site, and while it is mostly solventexposed the amine can interact favourably with the anionic Glu116 side chain. In chain $A$, in contrast, the aminopropyl moiety is disordered. Taken together, the intrinsic flexibility of the aminopropyl group, together with the observed conformational variability and its mostly solvent-exposed position, suggests that this functional group is less important for the binding of ispinesib to Eg5 than in other Eg5-targeting compounds which also contain this primary amine. This is further underlined by STLC and related analogues: in this case the primary amine is absolutely essential for $\mathrm{Eg} 5$ inhibition (Debonis et al., 2008) and an analogue with a tertiary amine completely abolishes inhibition, whereas a tertiary amine in ispinesib is still capable of inhibiting Eg5 in the low-nanomolar range (Sakowicz et al., 2004). It is remarkable that aside from the salt bridge between this primary amine and the Glu116 carboxylate, ispinesib makes exclusively hydrophobic interactions with the protein. In this context, it is particularly noteworthy that the carbonyl oxygen group of the quinazolin4-one ring system is buried in a hydrophobic pocket, which frustrates its hydrogen-bonding potential. While several potential weak $\mathrm{C}-\mathrm{H} \cdots \mathrm{O}$ hydrogen bonds are likely to at least partially compensate for this, the replacement of this group by a similarly sized hydrophobic group should provide improvements to $\mathrm{Eg} 5$ binding, although these modifications will have to be carefully balanced with its drug-like properties.

Although the unavailability of coordinates/structure factors precludes a detailed analysis, we felt it would be informative to compare our structure with the previously published Eg5ispinesib complex (Zhang et al., 2008). Overall, the two models<smiles>Cc1ccc(C(=O)N(CCCN)[C@H](c2nc3cc(Cl)ccc3c(=O)n2Cc2ccccc2)C(C)C)cc1</smiles>

Ispinesib

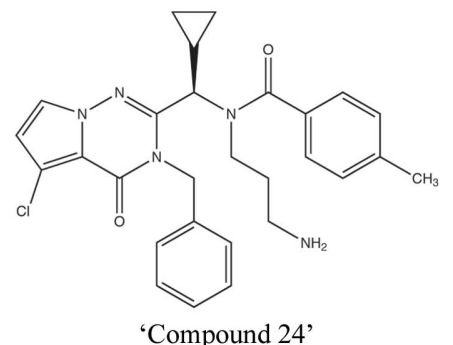<smiles>C[C@@H]1CN(C)CC[C@H]1N(C)C(=O)N1CC(c2cc(F)ccc2F)=C[C@@]1(CO)c1ccccc1</smiles>

MK-0731

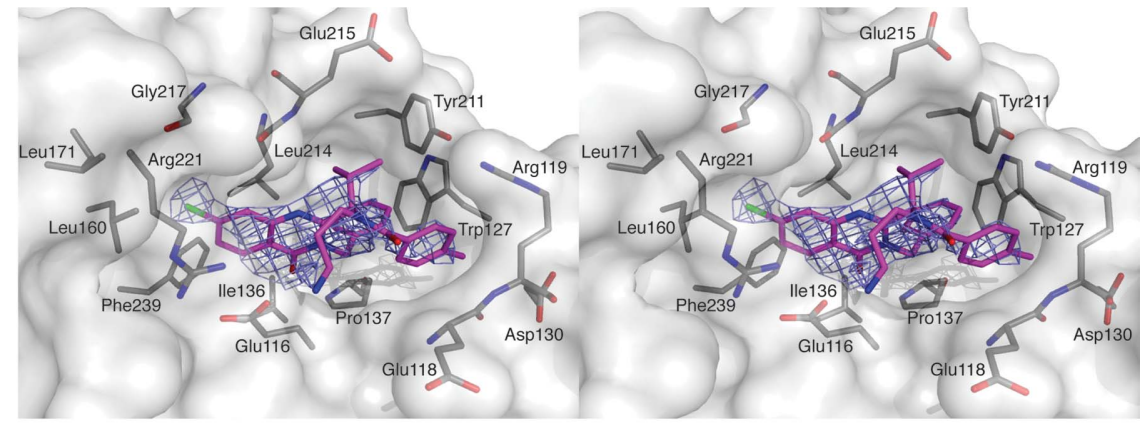

(a)

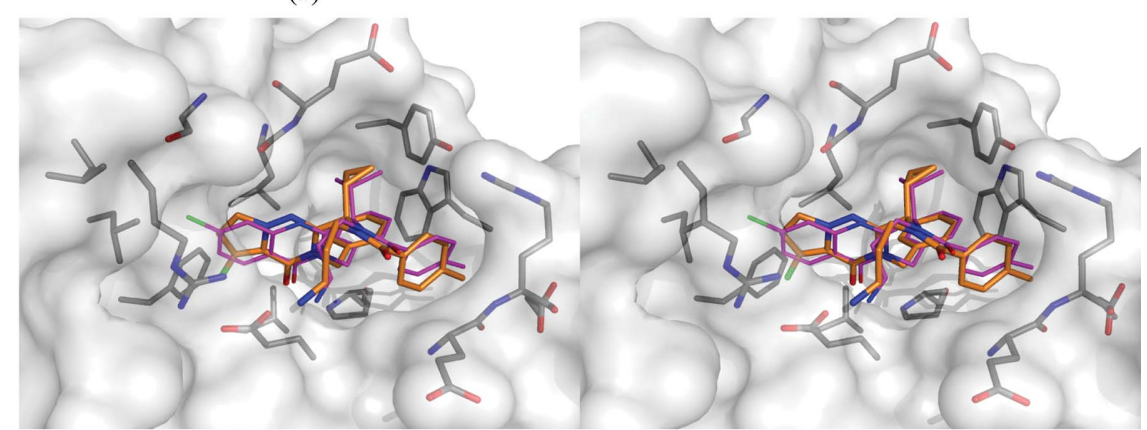

(b)

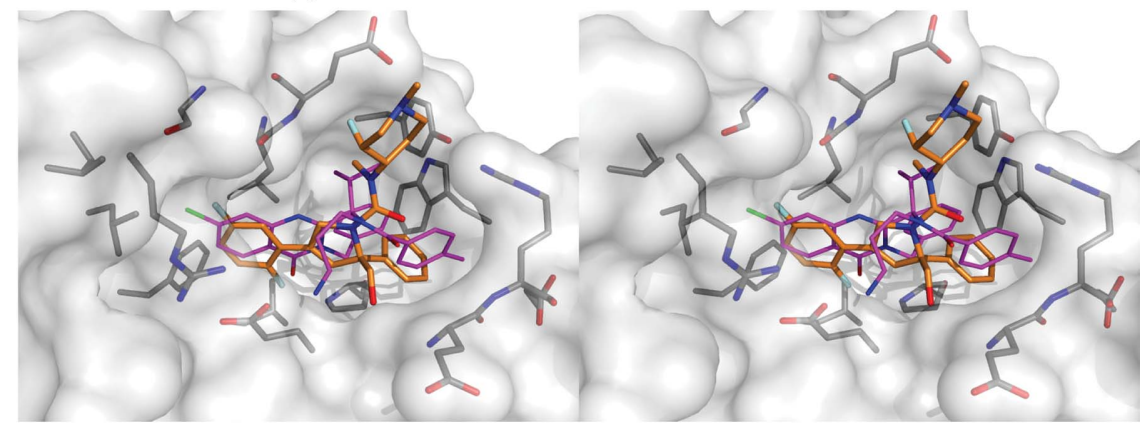

$(c)$

Figure 4

Interactions of ispinesib with the Eg5 inhibitor-binding region and comparison with other inhibitors of Eg5. (a) Chemical structure of ispinesib (left); stereoview of ispinesib (purple sticks) bound to the allosteric site of Eg5. Side chains and/or backbone atoms of interacting protein residues are shown as grey sticks and labelled; the protein surface is displayed semitransparently. Unbiased (i.e. calculated prior to including the ligand in the model) $\sigma_{\mathrm{A}}$-weighted $F_{\mathrm{o}}-F_{\mathrm{c}}$ electron density for the ligand contoured at $2.5 \sigma$ is shown in slate. (b) Chemical structure (left) and comparison of the Eg5 binding mode (right) of 'compound 24' (orange). (c) Chemical structure (left) and comparison of the Eg5 binding mode (right) of MK-0731 (orange). 
adopt a similar 'final state' conformation, although the previous structure exhibits a number of features that are unusual among published Eg5 structures and are not replicated by our model. Specifically, the helix between $\beta 1$ and $\beta 1$, as well as parts of the central $\beta$-sheet around $\beta 6 / \beta 7$, are shifted significantly with respect to the present complex structure. Additionally, loop 11 is ordered in the former structure, while in essentially all other Eg5 complex structures it is disordered. Zhang and coworkers suggest that these differences from the 'canonical' Eg5 conformation are a consequence of crystal contacts, which is plausible given their unique unit-cell parameters and is furthermore compatible with these features being absent from the present structure. Based on the discussion and figures provided by Zhang and coworkers, the conformation and binding mode of ispinesib appears to be virtually identical in the two structures, down to the orientation of the flexible aminopropyl group. While the isopropyl moiety of the ligand was apparently and unexpectedly modelled as a flat group in the previous structure, this has little effect on the observed ligand-protein interactions.

To further investigate the binding mode of ispinesib, we compared the structure of ispinesib-bound Eg5 with the Eg5-complex structures of two other related inhibitors: the pyrrolotriazin-4-one-based 'compound 24' (PDB entry 2gm1; Kim et al., 2006) and MK-0731 (PDB entry 2cjo; Cox et al., 2008) (Figs. $4 b$ and $4 c$ ).

Aside from the differences in the core ring system, 'compound 24 ' is virtually identical to ispinesib and it is thus not surprising that the two molecules adopt similar binding modes, with the benzyl, $p$-toluyl and aminopropyl groups all adopting consistent conformations and making equivalent interactions with the protein (Fig. $4 b$ ). The cyclopropyl group of compound 24 takes the place of the isopropyl group of ispinesib and again makes equivalent interactions. The most significant difference between these two compounds lies in the different attachment of the chlorine substituent to the core ring system, which is partly necessitated by the change from a quinazolinone (ispinesib) to a pyrrolotriazinone (compound 24). While the chlorine of ispinesib makes its closest contacts with the Leu160 side chain and the backbone of Gly217, the chlorine of compound 24 points deeper into the binding pocket (the angle between the two $\mathrm{C}-\mathrm{Cl}$ bonds after superposition is $\sim 104^{\circ}$ ), where it interacts predominantly with the side chains of Ile136 and Phe239. Given that the almost perfect alignment of the two core ring systems after superposition of the two complex structures on the protein component (cf. Fig. $4 b$ ) supports a lack of excessive strain owing to the presence of either chlorine, thus might suggest that the Eg5 affinity of either compound could be improved by introducing additional substitutions on the benzo and pyrrolo ring, respectively. At the same time, compound 24 is almost two orders of magnitude less potent as an Eg5 inhibitor compared with ispinesib (Kim et al., 2006), which might mean that it is compound 24 rather than ispinesib that would benefit most from such reciprocal elaboration.

While at first glance the 3-phenyl-dihydropyrrole-based MK-0731 shares few chemical features with ispinesib, a superposition of the two Eg5 complexes reveals the presence of several congruent structural elements (Fig. 4c). The difluorophenyl ring of MK-0731 takes the place of the quinazolinone system of ispinesib, with one fluorine mimicking the chlorine substituent of ispinesib and the second fluorine superimposing reasonably well with the quinazolone oxo group, although owing to the smaller size of F compared with $\mathrm{Cl}$ as well as the steric requirements of the rest of the molecule MK-0731 inserts less deeply into the binding pocket than does ispinesib. The unsubstituted phenyl ring of MK-0731 binds in the general area occupied by the chemically similar benzyl and $p$-toluyl groups of ispinesib. The reduced bulk and the positioning of this phenyl enable the side chain of Arg119 to close as a lid over this part of MK-0731, enabling favourable stacking interactions that are not accessible to ispinesib. A feature unique to MK-0731 is the hydroxymethyl group attached to the central dihydropyrrole ring. Introduced to increase polarity and thus reduce hERG binding (Cox et al., 2008), this group can be considered as related in purpose to the aminopropyl moiety of ispinesib, but its reduced intrinsic flexibility combined with local steric hindrance makes the hydroxymethyl group a potentially better choice that may inspire similar modifications in future ispinesib derivatives, although they will have to be monitored for possible phase II metabolic liabilities. Another feature unique to MK-0731 is its fluoromethylpiperidine 'side chain'. While it does interact with the protein, this moiety is mostly solvent-exposed and as such enables modulation of the physicochemical properties and thus the pharmacokinetics of the inhibitor. Despite the divergent binding features, ispinesib and MK-0731 are remarkably similar in their affinity for $\mathrm{Eg} 5$ (the $\mathrm{IC}_{50}$ of MK-0731 for MTstimulated Eg5 is $2 \mathrm{nM}$; Cox et al., 2008), again suggesting that 'transplanting' features may yield improved Eg5 ligands.

\subsection{Twinning and pseudotranslational symmetry}

Several data sets were collected from Eg5-ADP-ispinesib complex crystals, most of which could not be processed with any commonly used software. The present data could be indexed, processed and scaled in both primitive orthorhombic and $C$-centred monoclinic space groups. Structure solution by molecular replacement in these space groups was possible, yielding initially reasonable models, all of which subsequently failed to refine either because the $R$ values could not be decreased or because model completion revealed unavoidable clashes or otherwise impossible molecular arrangements. Decreasing the lattice symmetry to primitive monoclinic allowed the structure to be solved in space group $P 2_{1}$ for all three possible choices of crystallographic symmetry axis. Two of the three cell choices allowed model improvement through iterative refinement, while the third was yet again unrefinable. Inspection of the symmetry relationships between protein molecules in the $P 2_{1}$-refined models revealed three orthogonal twofold symmetry axes, two of which can individually function as crystallographic symmetry elements, while the third has a screw component of $\sim 0.38$ and as such provides only noncrystallographic symmetry. These findings explain the lack 
of refinement of the various orthorhombic models as well as one of the three $P 2_{1}$ axis choices, although it is somewhat surprising that believable models could be obtained from molecular replacement in these cases at all, as interpreting the 'odd' screw axis as a crystallographic $2_{1}$ symmetry axis would impose a coordinate shift of more than $13 \AA$ on parts of the model.

In addition, the $P 2_{1}$ model(s) exhibits pure translational (noncrystallographic) symmetry that almost, but not quite, mimics a face-centring operation, explaining why scaling and molecular replacement were successful in $C 2$ but again did not yield a refinable model. To add insult to injury, the Eg5ispinesib complex crystals appear to suffer from pseudomerohedral twinning, as suggested by the Britton plot (Britton, 1972), $H$-test (Yeates, 1997) and RvR plot (Lebedev et al., 2006), and supported by a significant drop in $R / R_{\text {free }}$ of over $7 \%$ when switching from untwinned to twinned refinement in PHENIX, with the twin law a twofold axis orthogonal to the crystallographic symmetry axis and a twin fraction of around 0.4 .

Both translational noncrystallographic symmetry and twinning complicate structure solution and even for the valid cell choices similar refinable but non-equivalent models were obtained from molecular replacement. The final structure presented here was selected based on refinement statistics, crystallographic packing and precedent for this cell choice and packing in the PDB in entry 2gm1 (Kim et al., 2006), the protein component of which was subsequently used as a search model for molecular replacement.

\section{Conclusion and biological significance}

Eg5 shows significant potential as a drug target for cancer chemotherapy and correspondingly has attracted widespread attention, with numerous inhibitors in various phases of drug development. All Eg5 inhibitors developed to date target the globular motor domain, where they bind to one of two distinct sites: ATP-competitive inhibitors bind either in or close to the ATP-binding site (P-loop; Luo et al., 2007; Parrish et al., 2007), whereas allosteric inhibitors bind to the L5 loop region. The allosteric Eg5 inhibitors are attractive not only because they avoid binding in competition with ATP/ADP, but more importantly because binding to the particularly long L5 loop of $\mathrm{Eg} 5$ provides these compounds with specificity over other closely related kinesins, which generally possess a much shorter L5 loop that cannot furnish a comparable binding pocket. Ispinesib is a promising allosteric Eg5 inhibitor that is currently in phase II clinical trials. It is thus surprising that no structural data for the Eg5-ispinesib complex have been available to date. The present structure of the ternary Eg5ADP-ispinesib complex seeks to remedy this. It shows that the ligand makes extensive hydrophobic, but essentially no hydrophilic, interactions with the allosteric binding pocket of Eg5. Analysis of the binding mode, as well as a comparison with other allosteric Eg5 inhibitors, suggests a number of ways in which ispinesib could be modified while either retaining or even improving its affinity for $\mathrm{Eg} 5$, an argument that is further supported by the development of the second-generation ispinesib-based analogue SB-743921 (Holen et al., 2011) and the amount of additional data on development of ispinesibrelated compounds in the patent literature (Matsuno et al., 2008). This information should prove invaluable for future iterations of this inhibitor scaffold, be it to improve potency, to alter the pharmacokinetics or to counter the ever-present threat of resistance (Jackson, 2005).

We thank the Diamond Light Source for access to beamline I03 (MX6683). This publication contains part of the doctoral thesis of SKT. We are grateful to Andrew Pannifer from the Drug Discovery Programme at the Beatson Institute for fruitful discussions and Cancer Research UK for financial support.

\section{References}

Adams, P. D., Grosse-Kunstleve, R. W., Hung, L.-W., Ioerger, T. R., McCoy, A. J., Moriarty, N. W., Read, R. J., Sacchettini, J. C., Sauter, N. K. \& Terwilliger, T. C. (2002). Acta Cryst. D58, 1948-1954.

Bergnes, G., Brejc, K. \& Belmont, L. (2005). Curr. Top. Med. Chem. 5, 127-145.

Blangy, A., Lane, H. A., d'Hérin, P., Harper, M., Kress, M. \& Nigg, E. A. (1995). Cell, 83, 1159-1169.

Britton, D. (1972). Acta Cryst. A28, 296-297.

Burris, H. A. III, Jones, S. F., Williams, D. D., Kathman, S. J., Hodge, J. P., Pandite, L., Ho, P. T., Boerner, S. A. \& Lorusso, P. (2011). Invest. New Drugs, 29, 467-472.

Chen, L. C., Rosen, L. S., Iyengar, T., Goldman, J. W., Savage, R., Kazakin, J., Chan, T. C. K., Schwartz, B. E., Abbadessa, G. \& Von Hoff, D. D. (2011). J. Clin. Oncol. 29(15_suppl), 3076.

Chen, V. B., Arendall, W. B., Headd, J. J., Keedy, D. A., Immormino, R. M., Kapral, G. J., Murray, L. W., Richardson, J. S. \& Richardson, D. C. (2010). Acta Cryst. D66, 12-21.

Cox, C. D. et al. (2008). J. Med. Chem. 51, 4239-4252.

Cox, C. D. \& Garbaccio, R. M. (2010). Anticancer Agents Med. Chem. 10, 697-712.

Debonis, S., Skoufias, D. A., Indorato, R. L., Liger, F., Marquet, B., Laggner, C., Joseph, B. \& Kozielski, F. (2008). J. Med. Chem. 51, 1115-1125.

Emsley, P. \& Cowtan, K. (2004). Acta Cryst. D60, 2126-2132.

Esaki, T., Seto, T., Ariyama, H., Arita, S., Fujimoto, C., Tsukasa, K., Kometani, T., Nosaki, K., Hirai, F. \& Yagawa, K. (2011). Arch. Drug Inf. 4, 23-31.

Good, J. A., Skoufias, D. A. \& Kozielski, F. (2011). Semin. Cell Dev. Biol. 22, 935-945.

Hackney, D. D. \& Jiang, W. (2001). Methods Mol. Biol. 164, 65-71.

Harrison, M. R., Holen, K. D. \& Liu, G. (2009). Clin. Adv. Hematol. Oncol. 7, 54-64.

Holen, K. D., Belani, C. P., Wilding, G., Ramalingam, S., Volkman, J. L., Ramanathan, R. K., Vasist, L. S., Bowen, C. J., Hodge, J. P., Dar, M. M. \& Ho, P. T. C. (2011). Cancer Chemother. Pharmacol. 67, 447-454.

Jackson, J. R. et al. (2005). Proceedings of the AACR-NCI-EORTC Molecular Targets and Cancer Therapeutics Meeting, abstract A253. Philadelphia: AACR.

Kaan, H. Y., Ulaganathan, V., Hackney, D. D. \& Kozielski, F. (2010). Biochem. J. 425, 55-60.

Kabsch, W. (2010). Acta Cryst. D66, 125-132.

Kapitein, L. C., Peterman, E. J., Kwok, B. H., Kim, J. H., Kapoor, T. M. \& Schmidt, C. F. (2005). Nature (London), 435, 114118.

Kim, K. S. et al. (2006). Bioorg. Med. Chem. Lett. 16, 39373942. 
Kozielski, F., DeBonis, S. \& Skoufias, D. A. (2007). Methods Mol. Med. 137, 189-207.

Lad, L., Luo, L., Carson, J. D., Wood, K. W., Hartman, J. J., Copeland, R. A. \& Sakowicz, R. (2008). Biochemistry, 47, 35763585.

Lebedev, A. A., Vagin, A. A. \& Murshudov, G. N. (2006). Acta Cryst. D62, 83-95.

Luo, L. et al. (2007). Nature Chem. Biol. 3, 722-726.

Matsuno, K., Sawada, J. \& Asai, A. (2008). Expert Opin. Ther. Pat. 18, 253-274.

Mayer, T. U., Kapoor, T. M., Haggarty, S. J., King, R. W., Schreiber, S. L. \& Mitchison, T. J. (1999). Science, 286, 971-974.

Miki, H., Setou, M. \& Hirokawa, N. (2003). Genome Res. 13, 14551465.

Murshudov, G. N., Skubák, P., Lebedev, A. A., Pannu, N. S., Steiner, R. A., Nicholls, R. A., Winn, M. D., Long, F. \& Vagin, A. A. (2011). Acta Cryst. D67, 355-367.

Parrish, C. A. et al. (2007). J. Med. Chem. 50, 4939-4952.

Sakowicz, R., Finer, J. T., Beraud, C., Crompton, A., Lewis, E., Fritsch, A., Lee, Y., Mak, J., Moody, R., Turincio, R., Chabala, J. C., Gonzales, P., Roth, S., Weitman, S. \& Wood, K. W. (2004). Cancer Res. 64, 3276-3280.
Schüttelkopf, A. W. \& van Aalten, D. M. F. (2004). Acta Cryst. D60, 1355-1363.

Sheth, P. R., Basso, A., Duca, J. S., Lesburg, C. A., Ogas, P., Gray, K., Nale, L., Mannarino, A. F., Prongay, A. J. \& Le, H. V. (2009). Biochemistry, 48, 11045-11055.

Souid, A. K., Dubowy, R. L., Ingle, A. M., Conlan, M. G., Sun, J., Blaney, S. M. \& Adamson, P. C. (2010). Pediatr. Blood Cancer, 55, 1323-1328.

Turner, J., Anderson, R., Guo, J., Beraud, C., Fletterick, R. \& Sakowicz, R. (2001). J. Biol. Chem. 276, 25496-25502.

Vaguine, A. A., Richelle, J. \& Wodak, S. J. (1999). Acta Cryst. D55, 191-205.

Weil, D., Garçon, L., Harper, M., Duménil, D., Dautry, F. \& Kress, M. (2002). Biotechniques, 33, 1244-1248.

Winn, M. D. et al. (2011). Acta Cryst. D67, 235-242.

Wordeman, L. (2010). Semin. Cell Dev. Biol. 21, 260-268.

Yan, Y., Sardana, V., Xu, B., Homnick, C., Halczenko, W., Buser, C. A., Schaber, M., Hartman, G. D., Huber, H. E. \& Kuo, L. C. (2004). J. Mol. Biol. 335, 547-554.

Yeates, T. O. (1997). Methods Enzymol. 276, 344-358.

Zhang, B., Liu, J.-F., Xu, Y. \& Ng, S.-C. (2008). Biochem. Biophys. Res. Commun. 372, 565-570. 